\title{
Alignment strategy for the ATLAS tracker
}

\section{Tobias Golling*}

Lawrence Berkeley National Laboratory ( $L B N L)$

E-mail: TFGolling@lbl.gov

The ATLAS experiment is a multi-purpose particle detector that will study high-energy particle collisions produced by the Large Hadron Collider. For the reconstruction of charged particles, and their production and their decay vertices, ATLAS is equipped with a sophisticated tracking system, unprecedented in size and complexity. Full exploitation of both the Inner Detector and the muon spectrometer requires an accurate alignment. The challenge of aligning the ATLAS tracking devices is discussed, and the ATLAS alignment strategy is presented and illustrated with both data and Monte Carlo results.

The 16th International Workshop on Vertex detectors September 23-28, 2007

Lake Placid, NY, USA

${ }^{*}$ Speaker. 


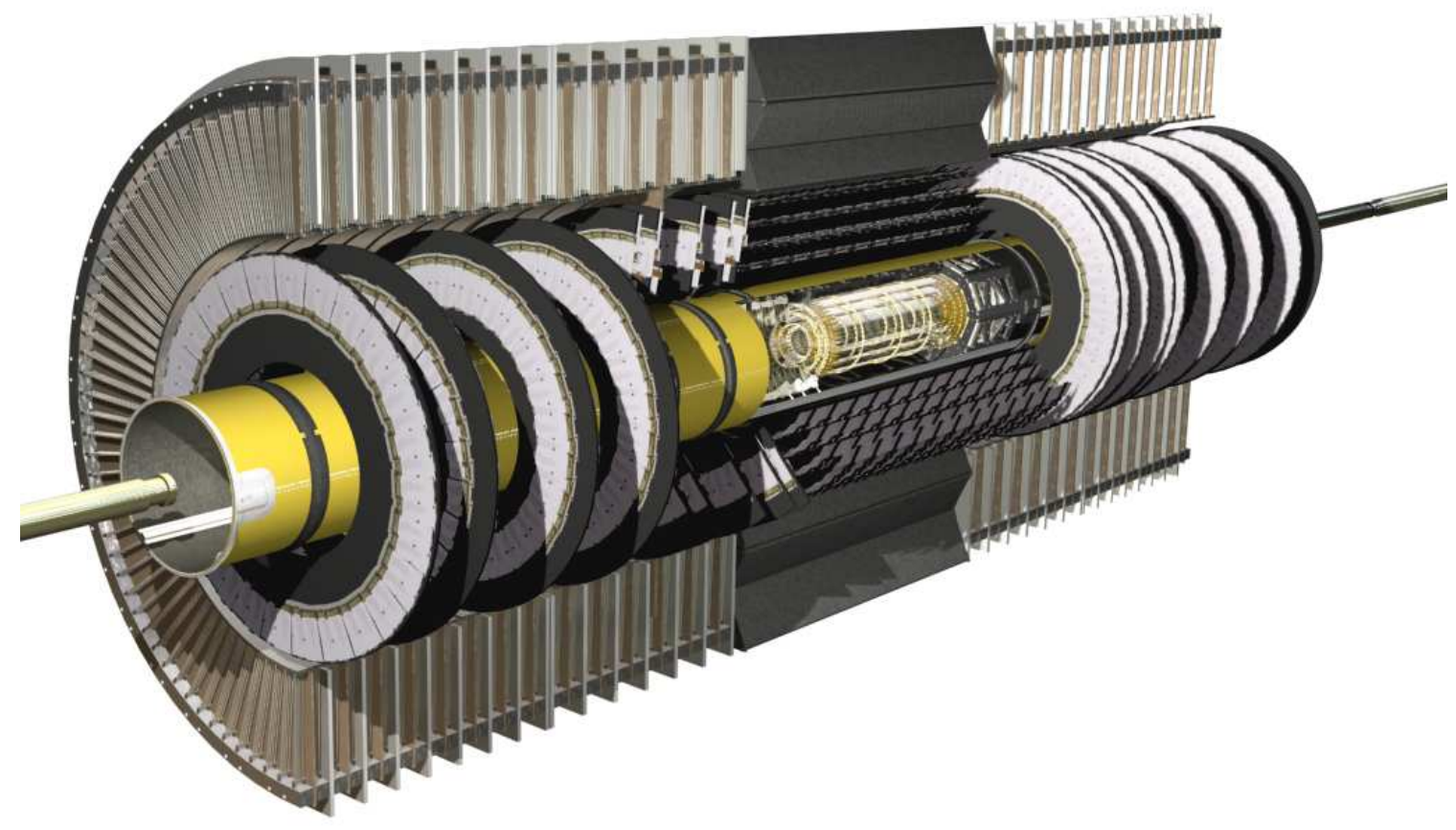

Figure 1: View of the ATLAS Inner Detector

\section{Introduction}

The ATLAS detector [1] is a general-purpose particle detector designed for high precision measurements of Standard Model processes and optimized for searches of the Higgs boson and New Physics, such as Supersymmetry or Extra Dimensions. The installation of the detector in situ is close to completion, hardware commissioning is ongoing in parallel, and ATLAS is expected to be ready for data taking in spring 2008 .

The ATLAS detector is composed of an Inner Detector [2], a calorimeter, and a muon spectrometer [3] capable of stand-alone track reconstruction. The Inner Detector is designed to measure the curved paths of charged particles in a magnetic field, and also particle production and decay vertices. Muons of momenta above a few $\mathrm{GeV}$ are also reconstructed in the muon spectrometer, since they are minimum ionizing particles and traverse the detector almost unperturbed. Both systems reconstruct particle four-momenta by position measurements which makes them sensitive to misalignments.

The ATLAS Inner Detector is composed of three complementary technologies designed to have an optimum performance of track reconstruction in the high-multiplicity high-frequency LHC (Large Hadron Collider) environment: a silicon pixel detector closest to the interaction point, followed by a silicon microstrip detector, and then the outermost straw-tube tracker, all surrounded by a superconducting solenoid providing a $2 \mathrm{~T}$ axial field. All three sub-detectors are composed of a barrel and two end-caps in the forward region (see Fig. 1). The pixel detector [4] is composed of three cylindrical barrel layers in the central region, and three disk layers in the forward region 
on either side. The layers consist of identical modules, with sensors segmented into 46080 pixels, each with an area of $50 \mu \mathrm{m} \times 400 \mu \mathrm{m}$, resulting in a resolution of $12 \mu \mathrm{m} \times 100 \mu \mathrm{m}$. The SCT (Semiconductor Tracker) [2] is made of four cylindrical barrel layers, and nine disks on each side. Modules are composed of two single-sided silicon microstrip sensors glued back-to-back with a relative stereo angle of $40 \mathrm{mrad}$ and a strip pitch of $80 \mu \mathrm{m}$, resulting in a resolution of $16 \mu \mathrm{m} \times 580 \mu \mathrm{m}$. The TRT (Transition Radiation Tracker) [2] consists of approximately $3 \times 10^{5}$ straw tubes arranged in three barrel layers and 14 end-cap wheels on each side. A resolution of $170 \mu \mathrm{m}$ is provided by a $30 \mu \mathrm{m}$ thick gold-plated tungsten wire at the center of each straw of $2 \mathrm{~mm}$ radius. Tracks reconstructed in the pseudorapidity region $|\eta|<2.5$ traverse at least three pixel layers and four SCT layers. For $|\eta|<2.1$ there are also 36 TRT measurements in the barrel region and a similar amount in the end-caps.

Exploitation of these excellent intrinsic resolutions necessitates a very precise alignment. The goal is to keep the degradation of the high $p_{T}$ track parameter uncertainties less than $20 \%$. This corresponds to an alignment precision of $10 \mu \mathrm{m}$ or better in the bending plane [2]. For a $W$ mass measurement the alignment uncertainty is the dominant source of systematics and the alignment precision needs to be improved to the one-micron level in order to get a precision on the $W$ mass of $25 \mathrm{MeV}$.

The ATLAS air-core muon spectrometer consist of about 1200 MDT (Monitored Drift Tube) chambers embedded in a toroidal magnetic field of $2 \mathrm{~T}$, surrounding the whole detector. Muons are reconstructed by three distinct MDT chamber planes up to $|\eta|<2.7$. The challenge is to measure the momentum of a $1 \mathrm{TeV}$ muon with a resolution of $10 \%$, which corresponds to measuring a $500 \mu \mathrm{m}$ sagitta with a $50 \mu \mathrm{m}$ accuracy. The single drift tube resolution is $80 \mu \mathrm{m}$, which translates to $40 \mu \mathrm{m}$ on measuring the sagitta. This defines the required alignment accuracy of $30 \mu \mathrm{m}$ to $40 \mu \mathrm{m}$.

The ATLAS strategy to reach these alignment goals is described in the following sections. Track-based alignment and its implementation in the ATLAS software is given in Section 2. There are shortcomings associated with the track-based alignment which can lead to a systematically misaligned detector geometry. Techniques are developed and discussed in Section 3 to control these systematics and to make the alignment more robust. Extensive validation of the alignment approach both with Monte Carlo simulation and with data is presented in Section 4. The alignment operation during collision data taking is outlined in Section 5. A summary and a conclusion is given in Section 6.

If not stated otherwise, coordinates refer to the global (right-handed) coordinate system of the detector, where the $z$-axis is parallel to the beam axis.

\section{Alignment algorithms}

Aligning a tracking detector such as ATLAS is a major endeavor. It is required to measure tens of thousands of parameters to a precision of a few microns. A nominal alignment is given by the detector design. However, the detector which is installed and will take data differs from its design. Reasons for that are the assembly precision, sagging due to gravity, deformations due to mechanical stresses or humidity. One needs to define the smallest detector element one wants to align. For example for the ATLAS pixel detector it is certainly unrealistic to try to obtain an 
alignment of each individual pixel, as this would correspond to hundreds of millions of parameters. For ATLAS the smallest detector elements subject to alignment are 1744 pixel modules (1456 of which in the barrel), 4088 SCT modules (2112 of which in the barrel), 96 TRT barrel modules, 28 TRT end-cap disks, and on the order of 1200 MDT chambers. Six alignment parameters are defined for each element, three for the position and three for the orientation in space. This corresponds to 34992 degrees of freedom for the silicon detectors alone. In addition to that modules can bow or twist which can can lead to a few more alignment parameters per detector element.

The alignment parameters can not be measured directly. However, the alignment corrections can be inferred from the fit quality of reconstructed tracks. This can be done by minimizing a $\chi^{2}$ function built from the sum of squared track residuals for a large number of tracks, where the residual is defined as the distance between the reconstructed hit and the intersection of the reconstructed track with the detector element:

$$
\chi^{2}=\sum_{\text {data sets }}\left(\sum_{\text {events }}\left(\sum_{\text {tracks }}\left(\sum_{\text {hits }} r^{T} V^{-1} r\right)\right),\right.
$$

where $r(a, \pi)$ is the vector of hit residuals which depends both on the alignment parameters $a$ and on the track parameters $\pi$, and $V$ is the covariance matrix of the hit measurements composed of the hit and the track uncertainties. Minimization of Eq. (2.1) leads to a very large (tens of thousands of parameters) system of equations. Several ways are pursued to solve the system, and all include various levels of approximations which can be recovered by iterative methods. Misalignments are small which allows a linear expansion from which the generic solution for the alignment correction reads:

$$
\delta a=-\left(\sum_{\text {all hits }} \frac{d r^{T}}{d a} V^{-1} \frac{d r}{d a}\right)^{-1}\left(\sum_{\text {all hits }} \frac{d r^{T}}{d a} V^{-1} r\right),
$$

where here and in the following the sum over all data sets, events, tracks and hits on tracks is abbreviated by the sum over all (these) hits.

\subsection{The Global $\chi^{2}$ algorithm}

The so-called Global $\chi^{2}$ algorithm [5] aims at solving the large system of equations of Eq. (2.2) by fully taking into account all correlations between different modules introduced by the reconstructed tracks traversing them:

$$
\frac{d r}{d a}=\frac{\partial r}{\partial a}+\frac{\partial r}{\partial \pi} \frac{d \pi}{d a}
$$

The solution of Eq. (2.2) represents a numerical challenge of inverting a symmetric matrix of size $N$, where $N$ is the number of degrees of freedom of the problem. Special care needs to be taken since the matrix is inherently singular, as the problem is under-constrained and can have more than one solution. The diagonalization of the matrix allows one to identify so-called "weakly determined modes" of the system (i.e. deformations of the detector which can not be easily distinguished) as the eigenvectors of the smallest eigenvalues. However, the diagonalization is very time consuming. Iterative solutions like fast sparse matrix techniques (MA27 [6]) require less storage and fewer operations, but do not have the reliability of the direct solution, and require preconditioning (see Section 4.1). 


\subsection{The Local $\chi^{2}$ algorithm}

The so-called Local $\chi^{2}$ approach [7] is based on the same ansatz (2.2), but the correlations between different detector elements are neglected. This is done by reducing the full derivative (2.3) to the partial derivative with respect to $a: \frac{d r}{d a} \rightarrow \frac{\partial r}{\partial a}$. As a consequence, the matrix $V$ reduces to a diagonal matrix and each degree of freedom $i$ is calculated independently:

$$
\delta a_{i}=-\left(\sum_{\text {all hits }} \frac{\partial r_{i}^{T}}{\partial a} \frac{1}{\sigma_{i}^{2}} \frac{\partial r_{i}}{\partial a}\right)^{-1}\left(\sum_{\text {all hits }} \frac{1}{\sigma_{i}^{2}} \frac{\partial r_{i}^{T}}{\partial a} r_{i}\right),
$$

where $\sigma_{i}$ is the uncertainty on the $i$ th degree of freedom. Inter-module correlations are restored by iteratively computing alignment constants, updating the detector geometry and re-fitting the tracks.

\subsection{The Robust algorithm}

The so-called Robust algorithm [8] is based on residual and overlap residual measurements. Full detector coverage is ensured by a design where modules overlap within a given layer. Overlap residuals are defined as the difference between two residuals from two such overlapping modules. The algorithm is based on centering track residual and overlap residual distributions in an iterative way, with focus on simplicity and robustness. With the current implementation the Robust method aligns only the translational degrees of freedom.

\section{Weakly determined degrees of freedom and how to constrain them}

As mentioned already in Section 2.1, track-based alignment inherently suffers from so-called "weakly determined modes" or "weak modes," which are deformations of the detector which alter the track parameters but do not (significantly) change the $\chi^{2}$ of the track. A simple example is aligning a detector element with tracks perpendicular to the detector element, which does not allow the determination of any out-of-plane movements from the track fit quality. More specifically for the barrel geometry of the ATLAS tracking devices there are weak modes of whole cylinders. Progressive rotations about the $z$-axis can lead to "clocking," introducing a $p_{T}$ bias which is opposite for positively and negatively charged particles. The "telescoping" mode is caused by layers progressively translated along the $z$-direction, as shown in the bottom left mode of Fig. 2. Similar modes exist for $x$ and $y$. A whole spectrum of high frequency weak modes exists, i.e. correlated translations and rotations of modules which result in systematic deformations of the whole detector; examples are depicted in Fig. 2. In the following sub-sections methods are discussed which can constrain these weak modes.

\subsection{Survey data and hardware alignment}

Relative positions and orientations of detector elements are precisely measured before the final installation. Fig. 3 shows an example of the measurement precision of surveying the pixel module positions on the disks, which is found to be of the order of one micron. The stability of the module positions is estimated by repeating the measurements from Fig. 3 after one month of extensive handling of the disks (including transporting, attaching tubes, removing and adding detector elements). No significant changes within the measurement precision were found. 

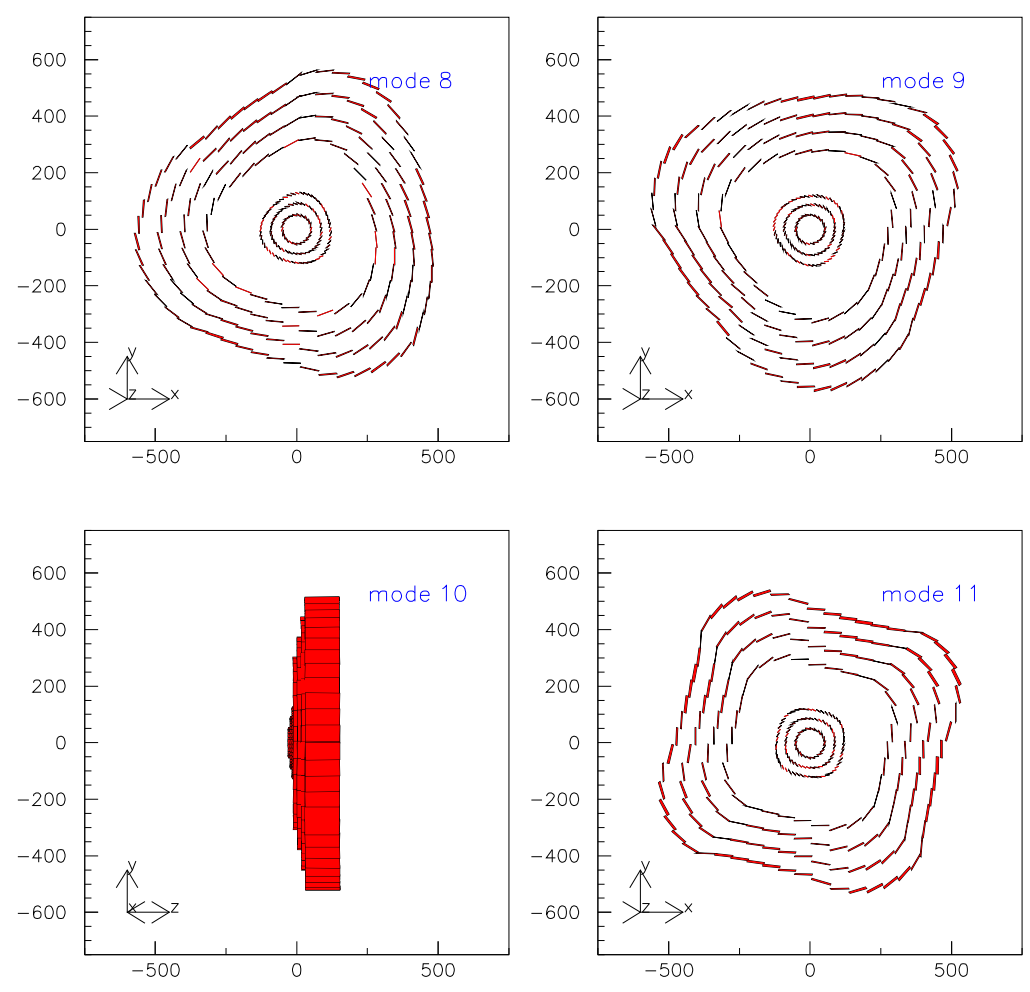

Figure 2: Some examples of lowest frequency weak modes, which approximately transform track helices into other helices. The contours show modules in the three pixel (inner) and the four SCT (outer) barrel layers: mode 8, 9 and 11 show projections of the modules onto the $x-y$-plane. Mode 10 shows the telescoping effect in a projection of all modules onto the $y$-z-plane. All units are in $\mathrm{mm}$. Distortions are exaggerated for better visibility.

This survey data is particularly useful to constrain relative positions and orientations of neighboring modules on rigid structures, by adding a $\chi^{2}$ penalty term corresponding to the survey precision and stability. Systematic effects due to changes in humidity and temperature are also estimated and taken into account. A method to incorporate survey data in alignment algorithms is given in [9].

The alignment of the ATLAS muon spectrometer is based on three different optical systems: Rasniks, BCAMs and Sacams [10]. The principle is the same for all three systems: an optical sensor looks through a lens towards a target and the sensor image is converted into translational and rotational alignment parameters. Some muon chambers are not optically linked. For these the alignment is complemented by track-based alignment with muons passing through overlap regions with optically linked chambers.

The SCT is equipped with a hardware alignment system, the so-called "Frequency Scanning Interferometry" (FSI), to resolve short term thermally induced distortions. A geodetic grid of length measurements between nodes attached to the SCT support structure measures 842 grid lines simultaneously. This can be repeated every 10 minutes with sub-micron precision and results in 

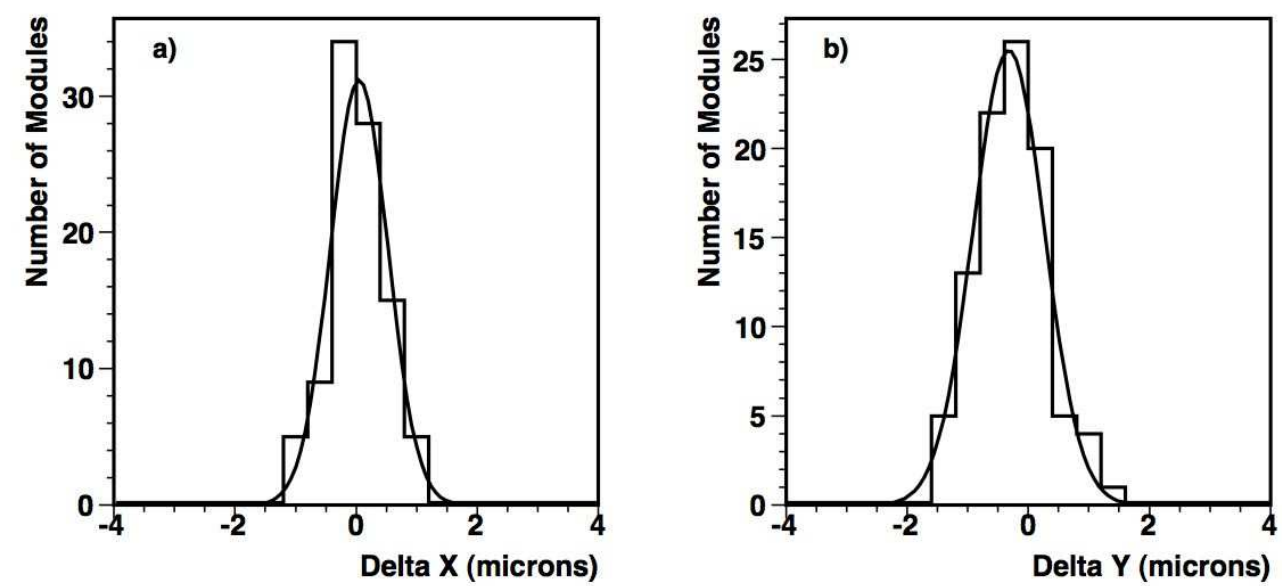

Figure 3: For two X,Y disk surveys taken one right after the other: a) difference in the $\mathrm{X}$ module alignment parameters, $\mathrm{b}$ ) difference in the $\mathrm{Y}$ module alignment parameters.

a quasi real-time tracking of shape changes. However, the system needs to be calibrated with an initial alignment, no absolute position measurement is possible, and one needs to model the correlation between FSI measurement and the actual module position.

\subsection{Physics constraints and detector redundancy}

A good complementary method to obtain alignment information is to use physics constraints, like a vertex constraint or tracks originating from a decay of a resonance. Tracks from $J / \Psi, \Upsilon$ or $Z$ resonances carry additional information (mass constraint, charge, forward-backward or $\phi$ symmetries) which is affected by alignment.

Position, energy and momentum of a charged particle are usually measured by more than one subsystem, like position and momentum measurement in the Inner Detector and the muon spectrometer, and position and energy measurement in the calorimeter. This redundancy can be used to impose additional constraints on the alignment algorithms.

\subsection{Other data sets: cosmic muons, halo muons and beam-gas}

The best performance of the alignment algorithms is given by combining various data sets that are sensitive to different types of misalignments. The robustness is improved by correlating as many modules as possible across the detector using different track topologies, including tracks which traverse both the barrel and the end-cap, and tracks giving overlap hits (see Section 2.3).

Cosmic muons are characterized by a very soft momentum spectrum and a rate which decreases strongly as the cosmic rays become more horizontal. They are the only tracks that link the top and the bottom hemispheres of the tracking detector. This unique property makes them invaluable, in particular to constrain the telescope mode. The ATLAS tracking devices are exposed to cosmic rays both on the surface before installation in their final position as well as in the pit before the start-up of LHC. The depth of the ATLAS detector $(100 \mathrm{~m})$ further decreases the rate 
of cosmic tracks usable for alignment, which is estimated in Monte Carlo simulation to be on the order of $1 \mathrm{~Hz}$ for tracks traversing the pixel detector with a momentum above a few GeV. It is very desirable to take cosmic events as well during collision data, given the unique properties of cosmic muon tracks. This can be done either in special runs in-between colliding beam operation or continuously with dedicated cosmic triggers during the actual proton-proton collisions. The feasibility of the latter is currently being investigated at ATLAS.

Already in single beam operation one can try to use tracks for alignment which are considered background in the colliding beam operation. Halo muons originate from beam halo protons which collide with the beam apertures, and produce a shower of secondary halo particles which eventually decay to muons. These halo muons are mainly parallel to the beam line but can have distances of a few meters in the transverse direction and can reach momenta close to the initial beam energy [11]. Halo muons are complementary to cosmic muons in the sense that they are the only tracks that link the forward to the backward region. A relative alignment of disks within an end-cap and of the the two end-caps with respect to each other is possible. Also beam-gas interactions provide tracks characterized by low momentum and being in the very forward region [11].

\section{Alignment validation before collision data}

The ATLAS alignment strategy is tested and validated both on Monte Carlo simulation, Section 4.1, and on data, Sections 4.2- 4.3.

\subsection{Alignment validation with Monte Carlo simulation}

The track-based alignment algorithms discussed in Section 2 can be tested for their robustness against misalignments before data comes available using Monte Carlo simulation. In particular the improvements that are discussed in Section 3 can be verified. This exercise is conducted within the so-called "Computing System Commissioning" (CSC) intended to exercise the full ATLAS software chain before data taking starts. A large sample of one million tracks is simulated with a misaligned detector setup, referred to as the "CSC misalignment." The introduced misalignment is based on the construction and assembly precisions: relative misalignments are on the order of one millimeter between the various subdetectors, and between 30 and 150 microns for individual modules. Two different event samples are utilized for the production of alignment constants: a non-physical sample of 10 muons per event originating from one common vertex with momenta between 2 and $50 \mathrm{GeV}$ (referred to as the "multi-muon" event sample), and cosmic ray events.

The alignment procedure for the silicon detector is performed in several iterations. Here the details are given for the Global $\chi^{2}$ algorithm (see Section 2.1): in a first step the barrel layers and end-cap disks are aligned with respect to each other. In a second set of iterations all individual modules are aligned with respect to each other. The sparse matrix techniques MA27 [6] is used. To guarantee robustness of the solution preconditioning is used by adding damping terms to each module degree of freedom according to the expected magnitude of the misalignment. This avoids alignment overcorrections. Alignment undercorrections are compensated by an iterative application of the algorithm. Repeating the first step of the alignment of individual layers at the end indicates that the layer to layer alignment is not altered by the individual module alignment. All 

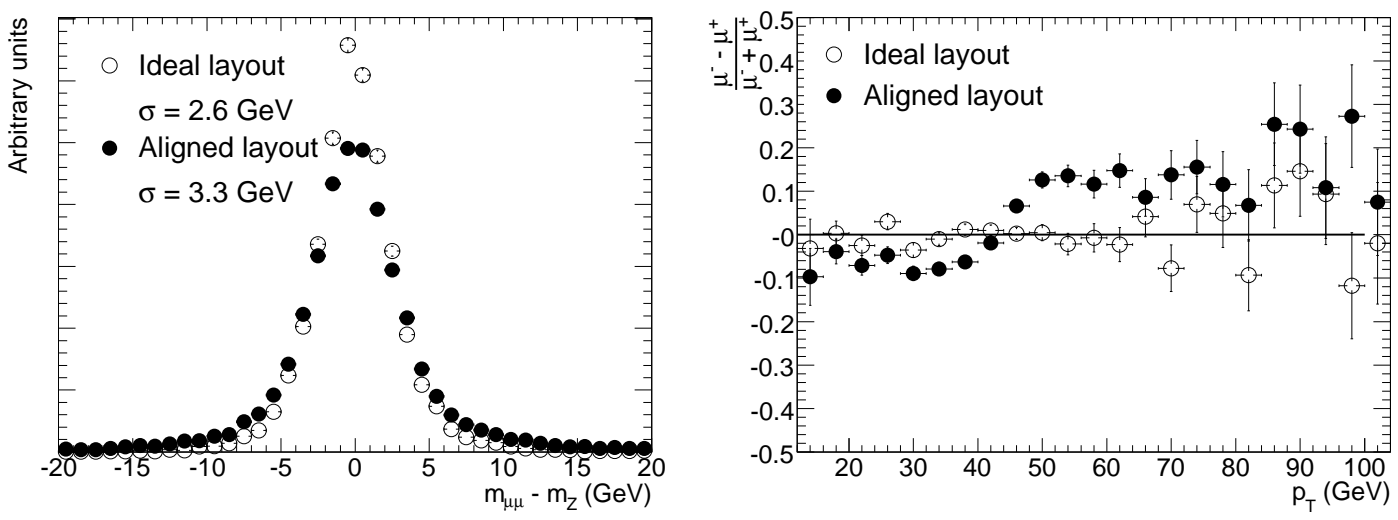

Figure 4: Left: difference between the reconstructed and the true mass of dimuon pairs from $Z \rightarrow \mu \mu$ events. Right: normalized difference of number of reconstructed negatively and positively charged muons from $Z \rightarrow \mu \mu$ events. Distributions are shown for both an ideal Inner Detector (perfectly aligned) and after the fi rst-pass alignment.

these iterations are performed with the multi-muon event sample. In addition a few thousand cosmic events are added in the last step which shows a considerable improvement in the reconstructed track parameters. After this internal silicon alignment the TRT modules are aligned with respect to the silicon detector. This first-pass alignment set is validated using various observables as described in more detail in Section 5.1. Fig. 4 shows the effect of residual misalignments on muon tracks, reconstructed only in the Inner Detector, from $Z \rightarrow \mu \mu$ events: the fitted Gaussian widths of the reconstructed $Z \rightarrow \mu \mu$ mass peaks are $2.6 \mathrm{GeV}$ for a perfectly aligned detector and $3.3 \mathrm{GeV}$ after the first-pass alignment. Fig. 4 also shows a charge- and $p_{T}$-dependent bias after the first-pass alignment, indicating the presence of a weak mode.

The underlying CSC misalignment contains a few weak modes (like e.g. a telescope mode) but lacks the presence of most of the other weak modes described in Section 3. It is foreseen to repeat this exercise with more pathological misalignments.

\subsection{Combined Test Beam (CTB) results}

All alignment algorithms are applied to the Combined Test Beam (CTB) data which was taken at the CERN $\mathrm{H} 8$ beam-test facility during 2004. Here only the silicon is subject to the alignment. The setup consists of 14 final prototype modules (6 pixel modules and 8 SCT modules) which are exposed to particles of various energies (between 2 and $180 \mathrm{GeV}$ ) and flavors (pions, electrons, muons), with and without a magnetic field of $1.4 \mathrm{~T}$ perpendicular to the beam axis. The incident angle is close to perpendicular to the module planes, and thus certain out-of-plane alignment constants are not determined.

In addition to the three algorithms discussed in Section 2 a fourth CTB-specific approach is added. All four algorithms give comparable results and agree very well with a Monte Carlo simulation of a perfectly aligned setup (except the Robust algorithm in Section 2.3, which has a slightly degraded performance due to the restrictions discussed there). Residual means are compatible with 

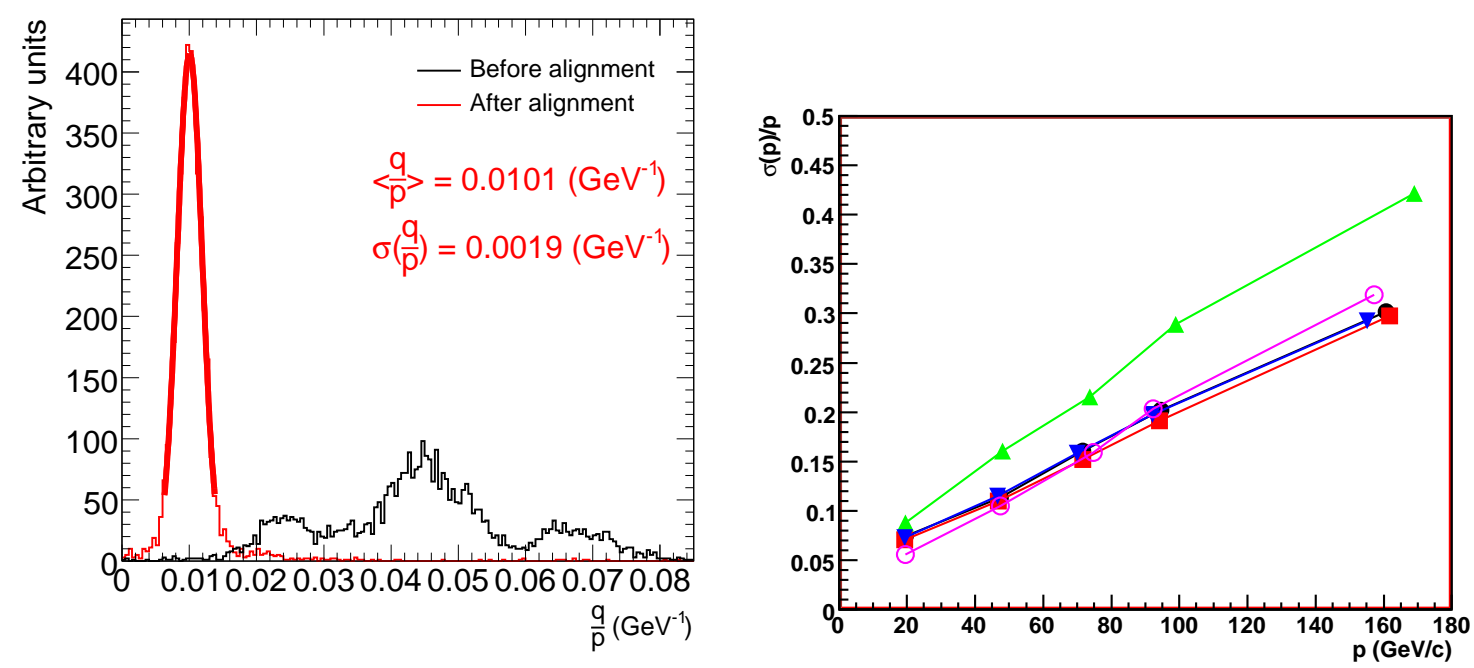

Figure 5: CTB results. Left: reconstructed curvature distribution for $100 \mathrm{GeV} \pi^{+}$with (red) and without (black) alignment corrections derived with the Local $\chi^{2}$ algorithm. Right: momentum resolution for electrons at various momenta for all four algorithms (Global $\chi^{2} \square$, Robust $\Delta$, Local $\chi^{2} \nabla$, CTB-specifi c $\boldsymbol{\bullet}$ ) and the Monte Carlo simulation $\bigcirc$. Only the Robust algorithm $(\Delta)$ deviates for reasons see text.

zero and the widths are as expected. A powerful means of validation is the proper reconstruction of track parameters. Figure 5 shows the curvature $(1 / p)$ distribution before and after the alignment corrections, and the momentum resolutions for the various algorithms for data sets with the magnetic field on.

\subsection{Cosmic muon results}

The ATLAS tracking devices are fully assembled on the surface. This allows one to operate and test them before they are installed in the ATLAS detector. Cosmic ray data were obtained with three separate setups in 2006: the combined SCT and TRT barrel, a combined SCT and TRT endcap, and a pixel end-cap. All three setups trigger on cosmics by placing scintillation counters above and below the detectors and requiring a coincidence. Low momentum muons $(<140-200 \mathrm{MeV})$ are rejected by a thick lead or concrete plate between the detector and the bottom scintillator. Other than that no momentum selection is possible since no magnetic field is present. As a consequence the momentum spectrum is dominated by low momentum muons and the residual resolution is dominated by multiple scattering.

All three algorithms are applied to the combined SCT and TRT barrel setup and show very good agreement for the residual distributions. Hit reconstruction efficiencies improve on average from $85 \%$ to $95 \%$. For the combined SCT and TRT end-cap the width of the residual distribution improves on average by $20 \%$ if the survey information is added to the nominal positions.

The pixel end-cap has only three layers which makes a track-based alignment difficult. However, it has the advantage of having about $24 \%$ overlap between modules in the same layer. This is used to derive alignment corrections between adjacent modules using both a pixel-specific algorithm and the Robust algorithm. Both algorithms get comparable results and agree well with a 

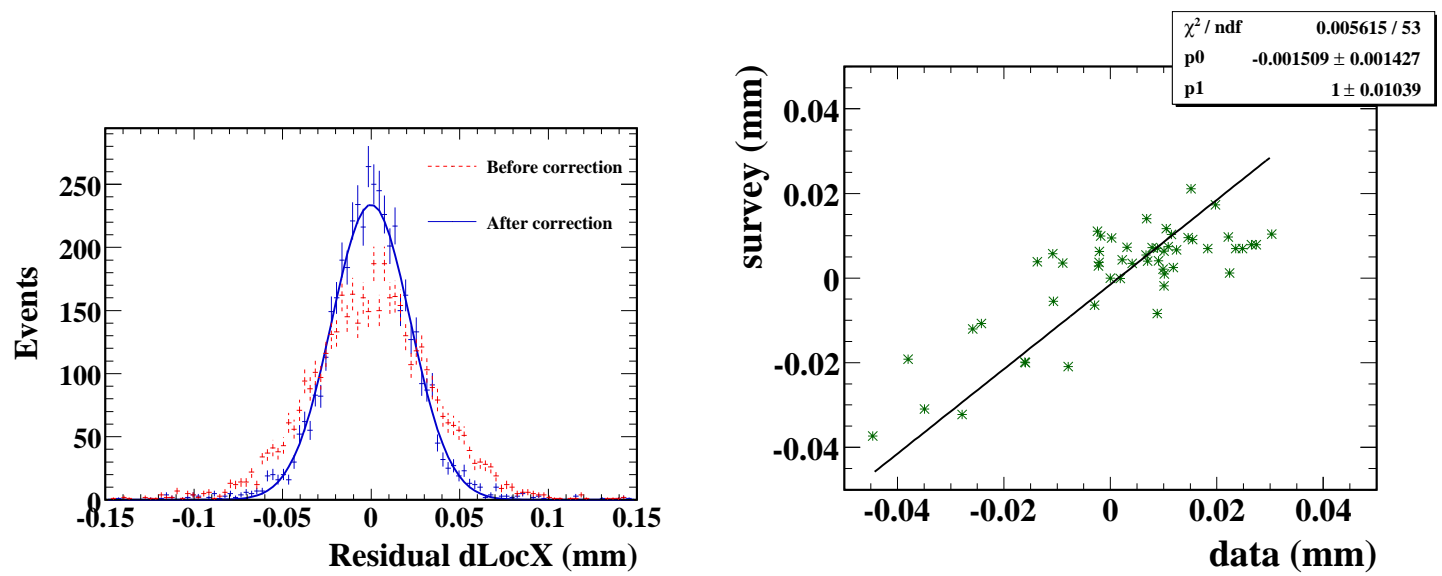

Figure 6: Left: residual distribution is shown in in the pixel end-cap using cosmic muons before and after the alignment corrections. Right: comparison of alignment corrections derived from the pixel-specifi c algorithm and from the survey.

dedicated Monte Carlo simulation. The residual distribution is shown in Fig. 6 before and after applying the alignment corrections from the pixel-specific algorithm. The width improves corresponding to a spatial resolution improvement in $x$ from $23 \mu \mathrm{m}$ to $16 \mu \mathrm{m}$, not far from the Monte Carlo expectation of $14 \mu \mathrm{m}$. In addition precise survey information exists for the pixel end-cap. A good correlation is seen in Fig. 6 between the alignment corrections and the survey data. More details can be found in [12].

The Inner Detector installation was completed on June 27, 2007. The detector subsystems are integrated into ATLAS in so-called "Milestone weeks," where ATLAS is operated and commissioned as a whole and cosmic data is recorded. The TRT already took part in Milestone week 4 (M4). The pixel and SCT are expected to be integrated and take cosmic ray data in spring 2008. A first alignment could be derived with cosmic muons before collision data taking starts.

\section{Alignment operation during collision data taking}

During collision data taking a dedicated Inner Detector alignment and calibration stream with a dedicated trigger menu is used to derive the alignment corrections for the ATLAS tracking devices. The alignment constants are validated before they are applied to the bulk reconstruction. For this a second so-called "express stream" is used. The alignment corrections are applied and the alignment quality is assessed by a monitoring tool. The timescale for deriving and validating the alignment of the Inner Detector is on the order of 24 hours.

This procedure will be tested in the so-called "Final Dress Rehearsal" where the full data processing chain is simulated and which will take place in 2008 before the LHC start-up.

\subsection{Alignment monitoring}

As mentioned in Section 3, alignment algorithms can converge and result in optimum residual distributions, however, "weak mode" misalignments might still be present. As a consequence, 

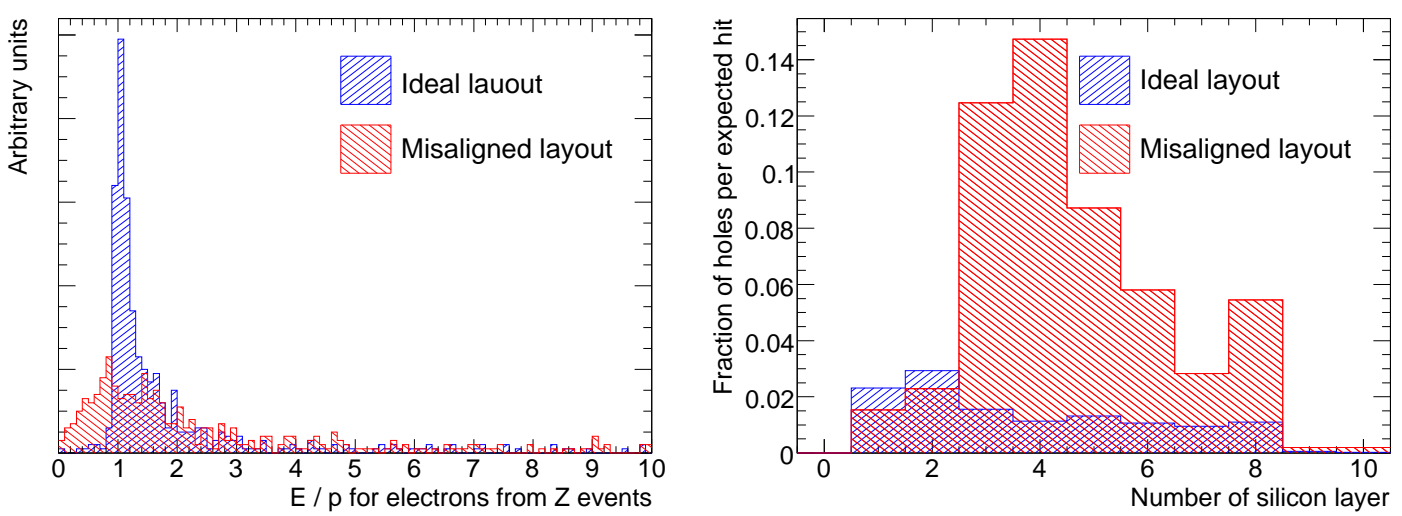

Figure 7: Left: $E / p$ distribution for a perfectly aligned detector (blue) and the CSC misalignment (red). Right: fraction of holes (a hole is defi ned as a missing hit in the sense that a reconstructed track intersects a sensitive detector element but no reconstructed hit is associated) per expected hit per detector layer ( 3 pixel layers and $4 \times 2$ SCT double layers), for a perfectly aligned detector (blue), and a particular weak mode (red).

a thorough validation is necessary which makes use of a well-defined and concise set of about 100 physics plots which have sensitivity to misalignments. Distributions can be compared to a reference either by eye or automatically, as shown for example in Fig. 7. The following categories of quantities are being monitored: hit efficiencies, generic track distributions, residual distributions, resonances, long lived particles, electrons, muons reconstructed in the muon spectrometer, cosmics, primary and secondary vertices and the reconstructed beamline.

In addition the alignment is expected to change with time. The monitoring can be used to assess the need for a reevaluation of alignment constants.

\section{Summary and conclusion}

The alignment of the ATLAS tracking devices is a difficult task. The ATLAS alignment strategy is based on track-based alignment and is complemented by hardware alignment, external constraints and physics constraints. The problem of such a large number of degrees of freedom benefits from a well-balanced choice of data sets to minimize systematic biases or local solutions. The more complementary the data sets are then the more robust the alignment determination will be. Three different algorithms are pursued. This software diversity allows to explore both traditional, simple and robust techniques, as well as novel, complicated and risky ones. Further improvements of the alignment strategy are focusing on the implementation of additional constraints and bootstrap methods.

The alignment strategy is tested with realistic deformation scenarios in a Monte Carlo simulation, data taken with a set of modules in a test beam, and cosmic data taken with individual subdetectors. Alignment exercises with Monte Carlo simulation show that the use of cosmic events greatly limits systematic biases. Preliminary alignment results with data are validated with sur- 
vey information. The alignment quality and time-dependent alignment effects are assessed by a dedicated alignment monitoring tool.

Dedicated data processing and storage of alignment constants is implemented. The focus is on an early preliminary alignment that is improved as more data is being collected. A first alignment of the whole tracking system with cosmic muons is expected in 2008 before the start of collision data taking.

\section{References}

[1] ATLAS Technical Design Report, Vol 1, CERN/LHCC/99-15 (1999).

[2] ATLAS Inner Detector Technical Design Report, CERN/LHCC/97-16 and CERN/LHCC/97-17 (1997).

[3] ATLAS Muon Spectrometer Technical Design Report, CERN/LHCC/97-22 (1997).

[4] ATLAS Pixel Collaboration, ATLAS Pixel Detector, Technical Design Report, CERN/LHCC/98-13 (1998).

[5] P. Brückmann de Renstrom et al., ATL-INDET-PUB-2005-002 (2005).

[6] I. S. Duff et al., Report R-10533, AERE Harwell Laboratory, Harwell UK (1982).

[7] R. Härtel, Diploma thesis, TU München, MPP-2005-174 (2005).

T. Göttfert, Diploma thesis, Universität Würzburg, MPP-2006-118 (2006).

[8] F. Heinemann, ATL-INDET-PUB-2007-011 (2007).

F. Heinemann, PhD Thesis, Univ. of Oxford, in preparation.

D. Hindson, PhD Thesis, Univ. of Oxford (2004).

[9] T. Golling, ATL-INDET-PUB-2006-001 (2006).

[10] S. Blusk et al., 'Proceedings of the fi rst LHC Detector Alignment Workshop," CERN Yellow Book 2007-004 (2007).

[11] M. Boonekamp et al., ATL-GEN-2004-001 (2004).

[12] ATLAS Pixel Collaboration, 'Pixel Offline Analysis for End-Cap A Cosmic Data," to be published. 